\title{
New Crestal Approach for Lifting Sinus in the Extremely Atrophic Upper Maxillae
}

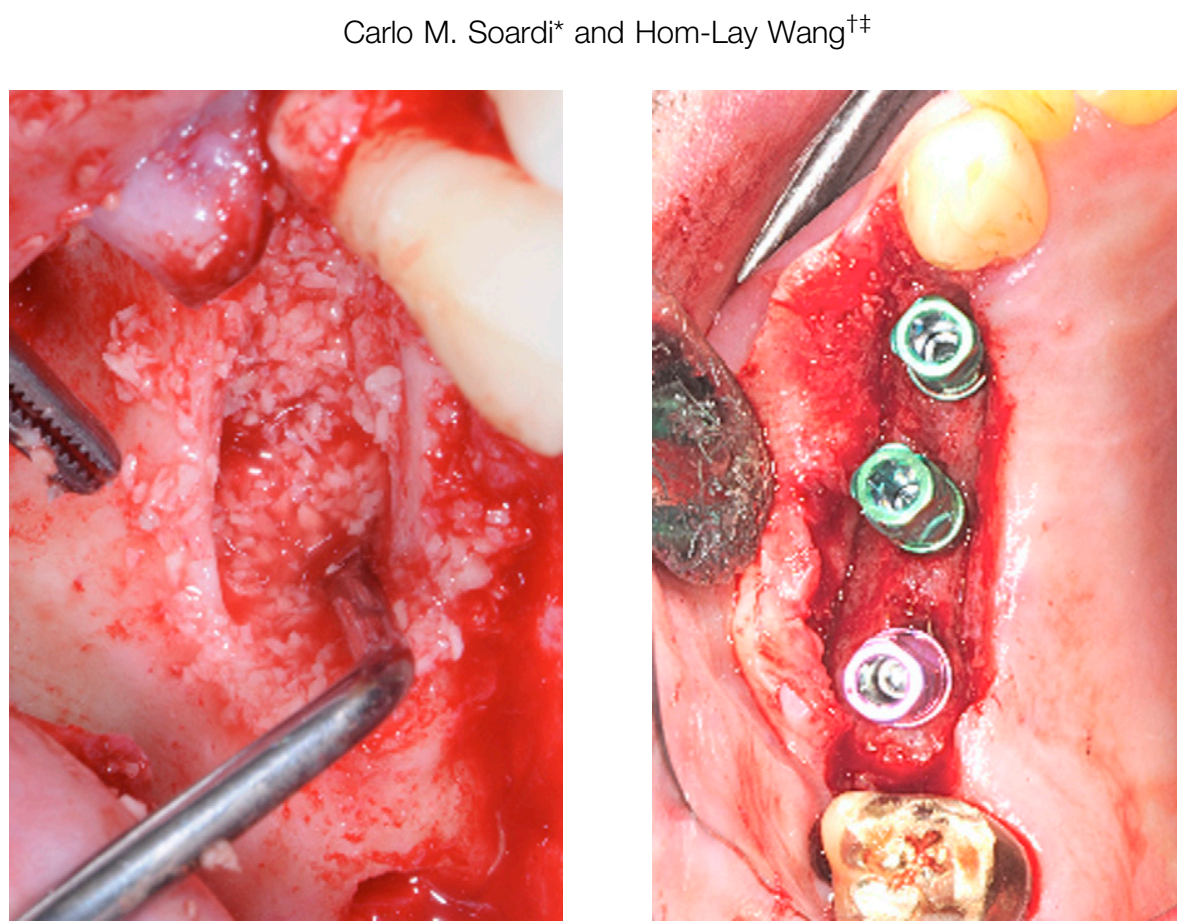

Introduction: Sinus augmentation, when performed with simultaneous or delayed implant placement, is a predictable procedure in augmenting deficient ridges. Traditionally, sinus augmentation was performed with either the lateral window or crestal osteotome approaches. In this case series, a new sinus floor elevation technique is proposed to treat specific cases of extremely atrophic maxillae.

Case Series: In this technique, a window is opened from the ridge crest instead of the lateral wall. Particulate bone graft is subsequently packed through the crestal window to elevate the Schneiderian membrane. Primary closure is achieved and implant placement is performed after healing is completed.

Conclusion: This new crestal sinus elevation technique combines direct visualization of the Schneiderian membrane with minimal flap reflection in the augmentation of severely atrophic maxillae in preparation for subsequent implant placement. Clin Adv Periodontics 2012;2:179-185.

Key Words: Bone regeneration; complications; dental implants; maxillary sinus; nasal mucosa.

\section{Background}

The maxillary sinus augmentation, first described by Tatum ${ }^{1}$ and subsequently modified by Boyne and James, ${ }^{2}$ is frequently indicated in patients with severe alveolar bone resorption or excessive pneumatization of the maxillary

\footnotetext{
* Private practice, Brescia, Italy.

' Department of Periodontics and Oral Medicine, School of Dentistry, University of Michigan, Ann Arbor, MI.

‡ College of Dentistry, King Saud University, Riyadh, Saudi Arabia.
}

Submitted August 10, 2011; accepted for publication October 8, 2011

doi: 10.1902/cap.2012.110076 sinuses, resulting in a vertical bone deficiency in the posterior maxilla. ${ }^{3}$ The surgical procedure involved elevating the Schneiderian membrane through a lateral window to create sufficient vertical space for bone augmentation in preparation for subsequent dental implant placement. Summers ${ }^{4}$ introduced an alternative surgical approach, which used a series of osteotomes that come in increasing diameters and are shaped with concave tips and sharpened edges to collect and condense bone in front of the advancing osteotome. In this technique, the maxillary sinus was accessed through the alveolar crest and peri-implant bone was simultaneously compacted, which was beneficial in sites with poorer bone quality, such as in the posterior maxilla. Dental implant placement could take place simultaneously 
with or after the augmentation. This depended on the ability to achieve primary implant stability, which typically was dictated by the amount of remaining alveolar bone. Maxillary sinus augmentation, achieved with either the lateral window or transcrestal approach, was fairly predictable and successful. Systematic reviews demonstrated an estimated annual implant failure rate of $2.5 \%$ to $3.5 \%$, a 3-year implant survival rate of $90 \%$ to $93 \%$ and a mean prevalence of membrane perforation of $3.8 \%$ to $19.5 \% .5,6$

In this case series, we propose a new crestal window approach that was a modification of the technique described by Winter et al. ${ }^{7}$ The main differences between these two techniques include: 1) a "beaver blade" was used to outline the lateral window in the technique of Winter et al., whereas a piezoelectric instrument ${ }^{\S}$ was used in our technique to create a larger crestal window, thus minimizing the risk of membrane perforation; 2) simultaneous implant placement was performed in the protocol by Winter et al., ${ }^{7}$ but we adopted a delayed implant placement approach because it was difficult to achieve primary implant stability predictably; and 3) absorbable collagen was used to stabilize the clot as well as maintain space in the protocol by Winter et al. ${ }^{7}$ compared to particulate grafts being placed in our protocol to maintain space and facilitate osteoconduction during healing. The purpose of this case series is to present this newly developed procedure.

\section{Clinical Presentation and Case Management}

Eight patients (six males and two females, aged 37 to 65 years), were enrolled in this case series. All patients needed sinus augmentations to receive implant-supported prostheses. A total of 14 maxillary sinuses that presented with extreme alveolar bone atrophy $(\leq 1 \mathrm{~mm}$ of residual vertical bone height) (Fig. 1a) were treated in private practice (Brescia, Italy) from July 2009 to June 2011 using the proposed transcrestal sinus lift approach described below. All patients provided oral consent to participate in this study.

\section{Incision}

A full-thickness crestal incision was made slightly toward the palate to maximize the chance of achieving primary wound closure (Figs. 1b and 2a). This incision should not be placed too palatally, because it would compromise blood supply to the site, leading to sloughing of the flaps and wound dehiscence. To facilitate access, a vertical or oblique release could be placed mesially and distally.

\section{Flap Elevation}

A conservative flap elevation, extending slightly beyond the alveolar crest, was performed to reduce flap morbidity. However, the reflection must be large enough to facilitate tension-free flap manipulation.

\section{Window Design}

A piezoelectric devicell was used to outline the window, so that the width of the window matched the width of the crest
(Figs. 1c and 2b). The depth of the osteotomy was determined from the thickness of the bone.

\section{Elevation of the Bony Window}

Once the crestal window was mobile, a piezoelectric tip was used to elevate the Schneiderian membrane away from the bony walls (Fig. 2c).

\section{Schneiderian Membrane Elevation}

This technique minimized membrane perforation by facilitating tension-free rapid detachment of the Schneiderian membrane (Figs. 1d and 2d). Thus, it might be beneficial around the septa in an attempt to minimize membrane perforation. The resultant contained defect whereby the roof was the mobilized alveolar crest would be an ideal site for bone regeneration. In cases of membrane perforation, a collagen membrane would be used to seal the perforation and prevent extravasation of the grafting material.

\section{Grafting Material}

For this technique, the use of particulated bone grafting material was suggested (Fig. 1d) but no particular material is strongly recommended. A collagen barrier membrane, which extended beyond the borders of the crestal window, was placed over the bone graft (Fig. 1e).

\section{Suture}

Primary wound closure was achieved using conventional suturing techniques (Fig. 1f). A postoperative cone-beam computed tomography (CBCT) scan showed the volume of bone graft placed in the sinus (Fig. 1g).

\section{Implant Placement and Second Stage}

After a 6-month healing period, a good amount of bone formation was observed at the surgical site (Fig. 1h). Three dental implants were then placed at prosthetically driven positions using surgical guides (Fig. 1i). An apically positioned flap was performed with the aim of increasing the zone of keratinized mucosa (Fig. 1j). A temporary prosthesis (e.g., three-unit splinted bridge) was delivered. Radiographic evaluation showed the amount of bone gain and increased bone density in grafted sinuses over 6 months (Fig. 1k).

\section{Radiograph}

A periapical radiograph was taken 6 months after implant placement to evaluate the marginal bone level.

\section{Clinical Outcomes}

Demographic information is summarized in Table 1. Initial bone thickness was not reported because it was $\leq 1 \mathrm{~mm}$. Adequate bone was formed in 13 of the 14 grafted sinuses, which made implant placement without additional grafting possible. A maximum of $1 \mathrm{~mm}$ of interproximal radiographic bone

\footnotetext{
$\S$ Mectron, Carasco, Italy

1 Mectron.

- Zimmer Dental, Carlsbad, CA.
} 

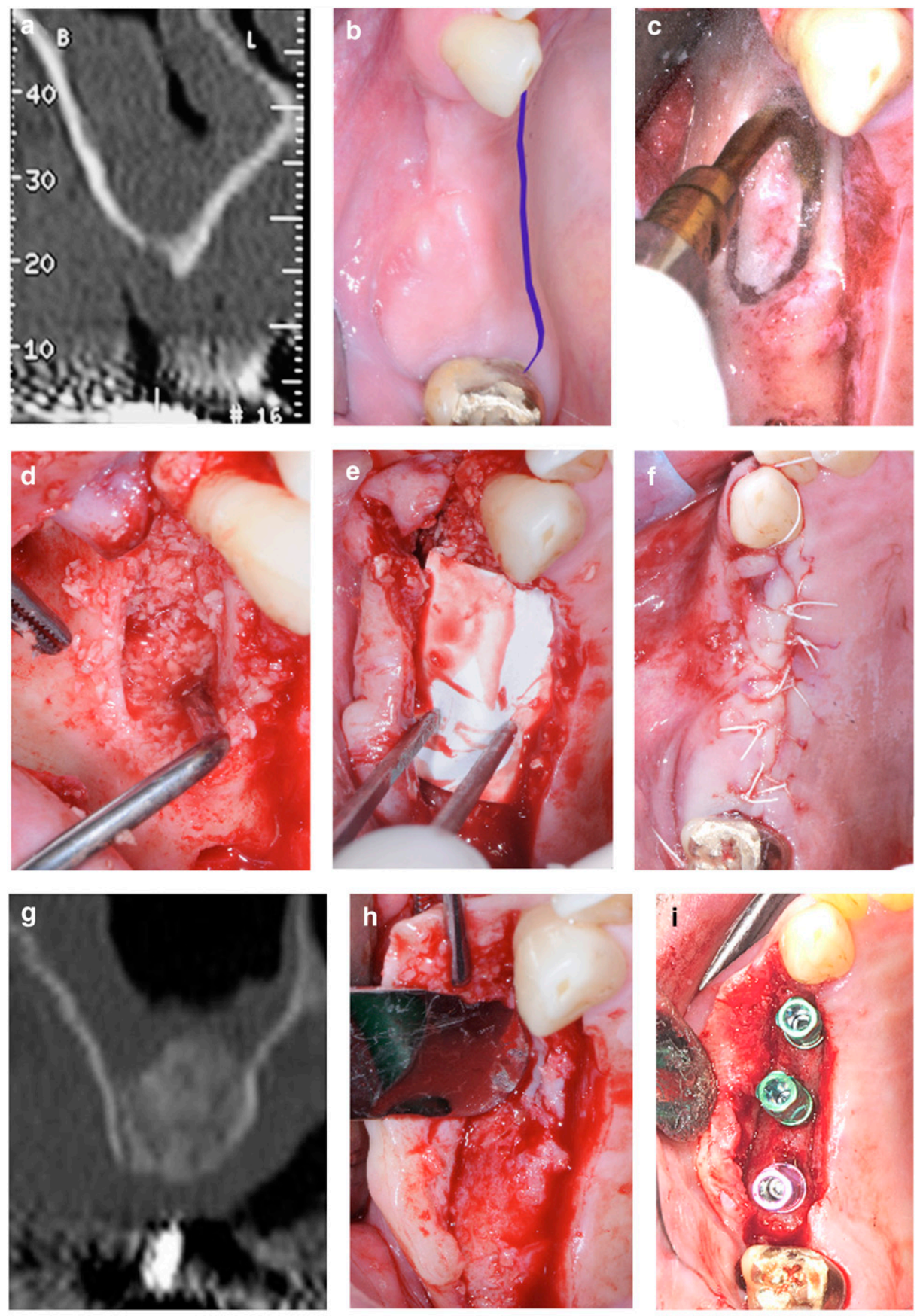

FIGURE 1a Preoperative CBCT scan showed minimal amount of bone available. 1b Incision design for crestal sinus lift approach. 1c Window opening using a piezoelectric instrument. 1d Lifting sinus membrane and placement of allergenic particulate bone grafts. 1e A collagen membrane was used to protect the bone graft and close the crestal window. If Tension-free flap closure achieved. 1g Postoperative CBCT scan showed amount of bone placed. 1h Sixmonth post-treatment showed bone formation around the surgical sites. 1i Three dental implants were placed using prosthetic-driven guides. $1 \mathrm{j}$ Flap was apically positioned so as to increase the zone of keratinized gingiva. 1k Six months after treatment, CBCT scan showed the large amount of bone regenerated.
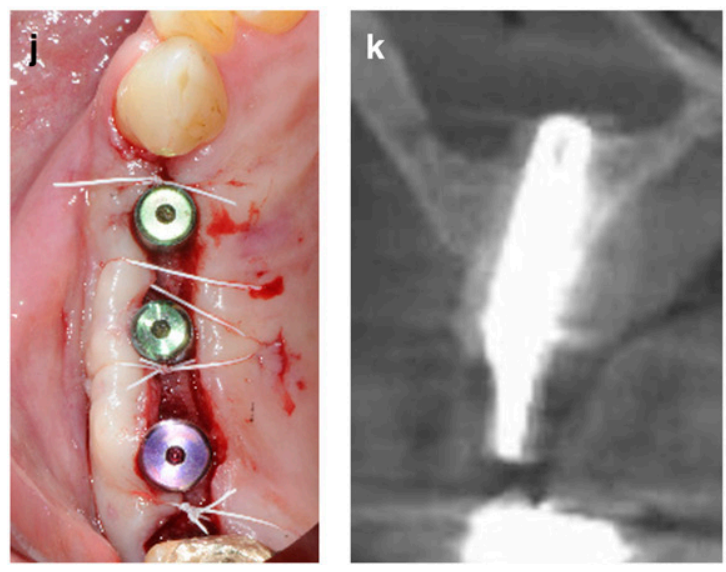
a

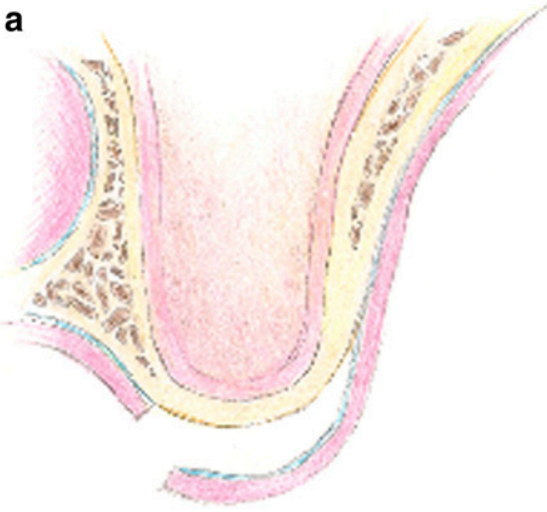

C

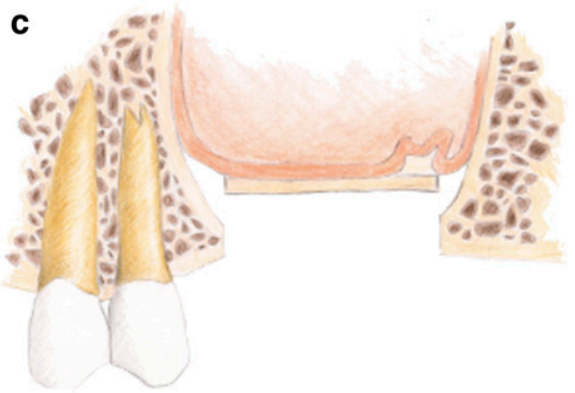

b

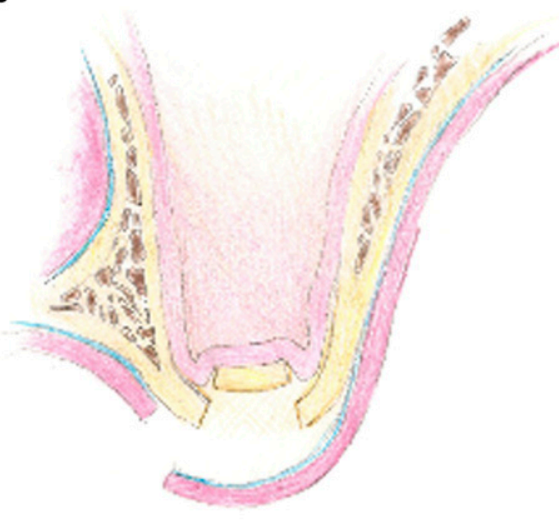

d

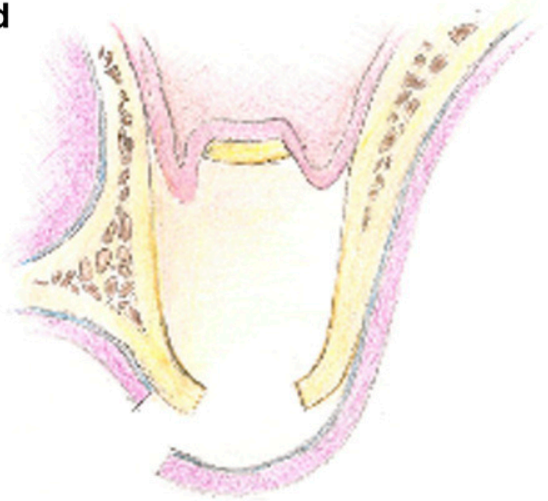

FIGURE 2 Drawing of crestal sinus lift technique. 2a A more palatally placed crestal incision design for the sinus crestal approach technique. 2b Window osteotomy outlined and Schneiderian membrane detached from the bony walls. $2 \mathrm{c}$ Tension-free detachment of the Schneiderian membrane. 2d Elevating the osteotomy window. resorption was noticed in 13 cases. Only one intraoperative complication, a Class 1 Schneiderian membrane perforation, ${ }^{8}$ was recorded.

One patient developed a significant postoperative complication, which was an oroantral communication of 5-mm diameter. This individual was a smoker, and the cause of the oroantral communication was speculated to be violent whistling, consequently leading to wound dehiscence, resulting in membrane exposure, infection, and partial loss of the bone graft. The patient was treated with antibiotic gels and a palatal obturator. Soft-tissue closure was observed 4 months later. A radiographic evaluation performed 6 months after sinus augmentation demonstrated bone regeneration, but it was not sufficient for implant placement. A second sinus lift using a lateral wall approach and simultaneous implant placement was then performed.

\section{Discussion}

Perforation of the Schneiderian membrane is the most common surgical complication when performing sinus augmentation. Neither transalveolar or lateral approaches guarantee the integrity of this anatomic structure. There is a higher risk of perforating the membrane when septa are present or in cases in which the membrane is thin. In addition, with the transalveolar approach, there is a higher risk of exceeding the elastic limits of the Schneiderian membrane during elevation, resulting in membrane perforation. ${ }^{9}$ This is because of the inability to control the amount of forces exerted on the osteotomes. Nonetheless, these problems can be minimized by using piezoelectric instruments. ${ }^{10}$ It is also difficult to directly visualize the sinus membrane through the osteotomy site, thus limiting the ability to identify a perforation. ${ }^{11}$ Conversely, the technique proposed in this case series offers both the advantages of direct visual control of the sinus membrane and characteristic of lateral approach techniques, and also the possibility to perform minimally invasive surgery.

Membrane perforation is highly associated with postoperative complications, ${ }^{12}$ which can manifest as sero-hematic nose effusion, grafting material extravasation, or acute/ chronic bacterial-induced sinusitis. ${ }^{12}$ The incidence of Schneiderian membrane perforation generally ranges from $7 \%$ to $35 \% .^{10,12}$ In this case series, a low incidence of membrane perforations $(6.67 \%)$ was observed. However, the results were preliminary and based on a limited population. In addition, the use of the piezo handpiece during the procedure might have helped to reduce the risk of membrane perforation. This technique allowed direct visualization of the sinus membrane because it was elevated through a conservative osteotomy, achieving a ridge height that was sufficient to place a $\geq 10$-mm-long implant (Table 1 ).

Compared to the lateral window approach, this technique might favor revascularization of the surgical site and minimize postoperative discomfort. This is because a more conservation flap was elevated, thus reducing surgical trauma. In addition, the risk of injuring the posterior alveolar artery or other vascular structures is diminished. Application of this technique has two main drawbacks. A second surgical procedure might be needed if the bone graft resorbed dramatically and a learning curve is associated with this 
TABLE 1 Demographic Information

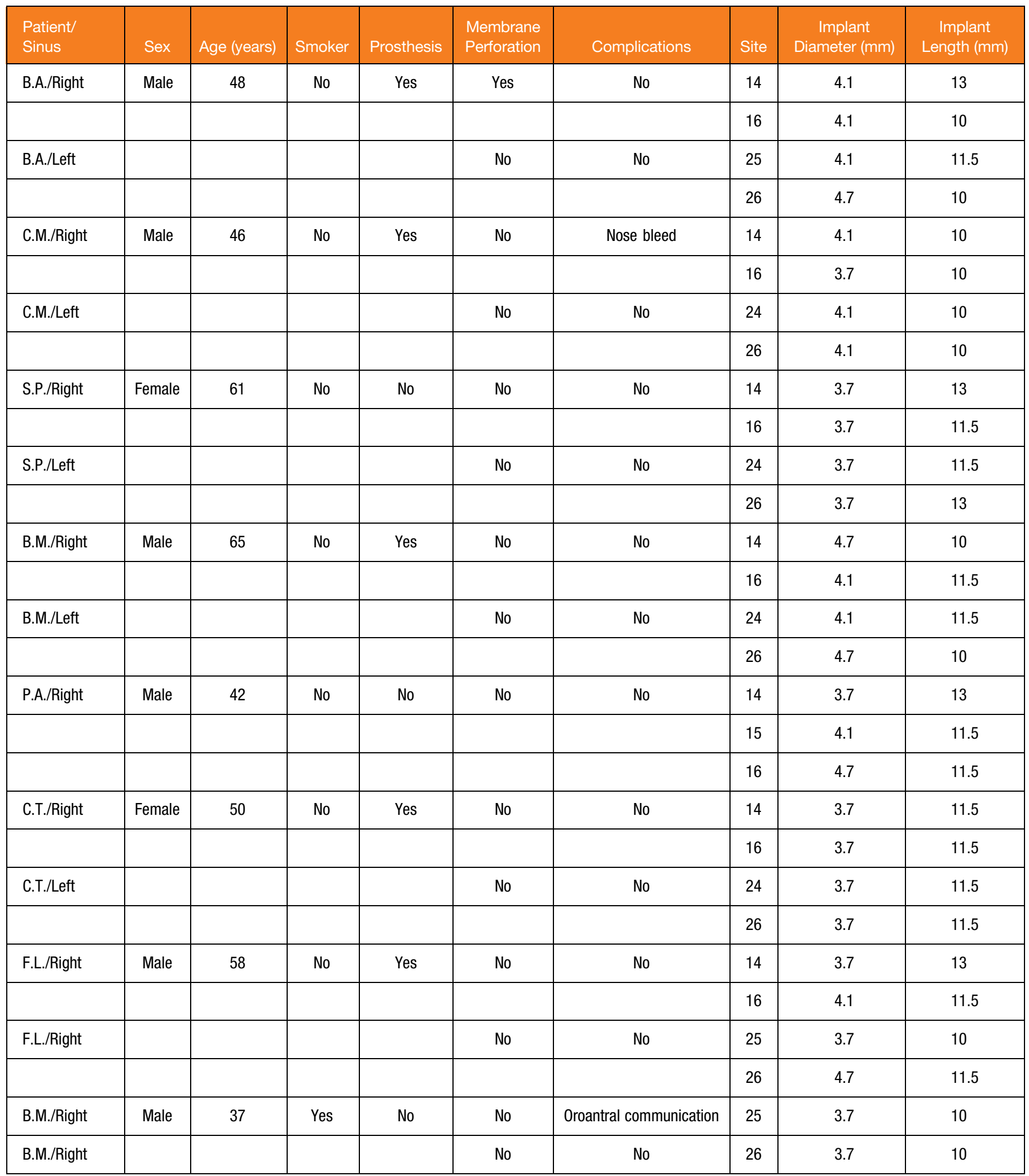

Total sinuses grafted: 14; total number of implants placed: 29; average length of implants placed: $11.26 \mathrm{~mm}$. 
technique. To reiterate, this technique aimed to obtain maximum regeneration with the least possible amount of trauma. Finally, this transcrestal technique achieved similar augmentation outcomes as those typically obtained by a lateral window approach.
This new crestal sinus elevation technique combines direct visualization of the Schneiderian membrane with minimal flap reflection in the augmentation of severely atrophic maxillae, in preparation for subsequent implant placement. However, future prospective studies are needed to validate this technique.

\section{Summary}

Why are these cases new information?
- This case series illustrated a new crestal approach that can predictably augment extremely atrophic maxillae and minimize postoperative complications.

- The keys to successfully managing these cases were primary wound closure and no sinus membrane perforation.

- The primary limitations were perforation of the sinus membrane and inadequate bone augmentation, resulting in a need for a second surgical procedure in one patient.

\section{Acknowledgments}

The authors thank Davide Zaffe (Professor, Department of Anatomy and Histology, University of Modena and Reggio Emilia, Modena, Italy) and Dr. Sergio Spinato (private practice, Sassuolo, Italy) for their selfless and essential collaboration in the development of this clinical study. In addition, the authors acknowledge Drs. Gustavo Avila (Assistant professor, University of Iowa, Iowa City, Iowa) and Jia-Hui Fu (graduate student, University of Michigan, Ann Arbor, Michigan) for their assistance in editing this paper and Barbara Soardi (Brescia, Italy) for the illustrations. Dr. Wang has received lecture fees and financial support for research from Zimmer Dental (Carlsbad, California). Dr. Soardi reports no conflicts of interest related to this case series.
CORRESPONDENCE:

Dr. Hom-Lay Wang, Department of Periodontics and Oral Medicine, University of Michigan, School of Dentistry, 1101 N. University Ave., Ann Arbor, MI 48109-1078. E-mail: homlay@umich.edu. 


\section{References}

1. Tatum H Jr. Maxillary and sinus implant reconstructions. Dent Clin North Am 1986;30:207-229.

2. Boyne PJ, James RA. Grafting of the maxillary sinus floor with autogenous marrow and bone. J Oral Surg 1980;38:613-616.

3. Smiler DG, Johnson PW, Lozada JL, et al. Sinus lift grafts and endosseous implants. Treatment of the atrophic posterior maxilla. Dent Clin North Am 1992;36:151-186; discussion 187-188.

4. Summers RB. The osteotome technique: Part 3 - Less invasive methods of elevating the sinus floor. Compendium 1994;15:698, 700, 702-704 passim; quiz 710 .

5. Tan WC, Lang NP, Zwahlen M, Pjetursson BE. A systematic review of the success of sinus floor elevation and survival of implants inserted in combination with sinus floor elevation. Part II: Transalveolar technique. J Clin Periodontol 2008;35(Suppl. 8):241-254.

6. Pjetursson BE, Tan WC, Zwahlen M, Lang NP. A systematic review of the success of sinus floor elevation and survival of implants inserted in combination with sinus floor elevation. I Clin Periodontol 2008;35 (Suppl. 8):216-240.

indicates key references.
7. Winter AA, Pollack AS, Odrich RB. Sinus/alveolar crest tenting (SACT): A new technique for implant placement in atrophic maxillary ridges without bone grafts or membranes. Int J Periodontics Restorative Dent 2003;23:557-565.

8. Vlassis JM, Fugazzotto PA. A classification system for sinus membrane perforations during augmentation procedures with options for repair. $I$ Periodontol 1999;70:692-699.

9. Pommer B, Unger E, Sütö D, Hack N, Watzek G. Mechanical properties of the Schneiderian membrane in vitro. Clin Oral Implants Res 2009; 20:633-637.

10. Wallace SS, Mazor Z, Froum SJ, Cho SC, Tarnow DP. Schneiderian membrane perforation rate during sinus elevation using piezosurgery: Clinical results of 100 consecutive cases. Int I Periodontics Restorative Dent 2007;27:413-419.

11. Nkenke E, Schlegel A, Schultze-Mosgau S, Neukam FW, Wiltfang J. The endoscopically controlled osteotome sinus floor elevation: A preliminary prospective study. Int J Oral Maxillofac Implants 2002;17:557-566.

12. Hernández-Alfaro F, Torradeflot MM, Marti C. Prevalence and management of Schneiderian membrane perforations during sinus-lift procedures. Clin Oral Implants Res 2008;19:91-98. 